\title{
Second messenger-mediated spatiotemporal control of protein degradation regulates bacterial cell cycle progression
}

\author{
Anna Duerig, ${ }^{1}$ Sören Abel, ${ }^{1}$ Marc Folcher, ${ }^{1}$ Micael Nicollier, ${ }^{1}$ Torsten Schwede, ${ }^{1}$ Nicolas Amiot, ${ }^{2}$ \\ Bernd Giese, ${ }^{2}$ and Urs Jenal ${ }^{1,3}$ \\ ${ }^{1}$ Biozentrum, University of Basel, 4056 Basel, Switzerland; ${ }^{2}$ Department of Chemistry, University of Basel, 4056 Basel, \\ Switzerland
}

\begin{abstract}
Second messengers control a wide range of important cellular functions in eukaryotes and prokaryotes. Here we show that cyclic di-GMP, a global bacterial second messenger, promotes cell cycle progression in Caulobacter crescentus by mediating the specific degradation of the replication initiation inhibitor CtrA. During the G1-to-Sphase transition, both CtrA and its cognate protease ClpXP dynamically localize to the old cell pole, where CtrA is rapidly degraded. Sequestration of CtrA to the cell pole depends on PopA, a newly identified cyclic di-GMP effector protein. PopA itself localizes to the cell pole and directs CtrA to this subcellular site via the direct interaction with a mediator protein, RcdA. We present evidence that c-di-GMP regulates CtrA degradation during the cell cycle by controlling the dynamic sequestration of the PopA recruitment factor to the cell pole.

Furthermore, we show that cell cycle timing of CtrA degradation relies on converging pathways responsible for substrate and protease localization to the old cell pole. This is the first report that links cyclic di-GMP to protein dynamics and cell cycle control in bacteria.
\end{abstract}

[Keywords: PopA; cyclic di-GMP; protein degradation; Caulobacter crescentus; cell cycle; second messenger]

Supplemental material is available at http://www.genesdev.org.

Received August 13, 2008; revised version accepted November 7, 2008.

Regulated proteolysis has a major impact on cellular physiology as it plays a primordial role in cell cycle control, stress response, and cell differentiation in both prokaryotes and eukaryotes. To avoid unwanted protein damage, eukaryotic cells largely restrict proteolysis to specific cellular compartments. In bacteria, several energy-dependent cytoplasmic proteases and their associated factors are responsible for the rapid degradation of several key cellular regulators (Gottesman 2003; Jenal and Hengge-Aronis 2003). These so-called selfcompartmentalizing proteases select their substrates through an ATPase complex, which gates the access to the proteolytic active site. To specifically select target proteins destined for degradation, bacteria have evolved a series of regulatory mechanisms, including transtranslation (Keiler et al. 1996), interference of small effector molecules (Zhu and Winans 2001), preprocessing (Alba et al. 2002; Kanehara et al. 2002), protein associa-

${ }^{3}$ Corresponding author.

E-MAIL urs.jenal@unibas.ch; FAX 41-61-267-2118.

Article is online at http://www.genesdev.org/cgi/doi/10.1101/gad.502409. tion (Johansson and Uhlin 1999; Gonzalez et al. 2000), or the use of specific targeting factors (Turgay et al. 1998). The recent observation that in Caulobacter crescentus the master cell cycle regulator CtrA dynamically sequesters to the old cell pole, where it is degraded by the polar ClpXP protease complex, suggested that spatial concurrence might also play a role in protein degradation control in bacteria (Iniesta et al. 2006; McGrath et al. 2006).

In C. crescentus, protein degradation plays an important role in controlling cell cycle progression (Domian et al. 1997; Jenal and Fuchs 1998; Grünenfelder et al. 2001). Caulobacter cells divide asymmetrically to produce two distinct daughter cells, a smaller motile swarmer cell and a larger surface-adherent stalked cell. Whereas the newborn stalked cell enters $S$ phase and reinitiates chromosome replication immediately, the chromosome of the swarmer cell remains quiescent for an extended period, equivalent to the G1 phase of eukaryotic cells. Concurrent with the morphological transformation of the swarmer cell into a stalked cell, the replication block is suspended, and cells proceed into $S$ phase. Differential activity of the essential response regulator CtrA is critical 
to control the Caulobacter G1-to-S-phase transition. Phosphorylated CtrA, CtrA 2P, blocks the initiation of replication by directly binding to five sites in the chromosomal OriC region, where it apparently restricts access of replication initiation factors (Quon et al. 1998). The activity of CtrA is redundantly controlled at the levels of expression, phosphorylation, and degradation (Domian et al. 1997, 1999). To initiate chromosome replication, activated CtrA $\sim \mathrm{P}$ is eliminated from the cell by two redundant mechanisms, temporally controlled dephosphorylation and proteolysis (Domian et al. 1997).

In vivo and in vitro experiments have demonstrated that the essential ClpXP protease complex degrades CtrA during G1-to-S transition (Jenal and Fuchs 1998; Chien et al. 2007). Intriguingly, the ClpXP protease complex transiently sequesters to the incipient stalked cell pole during the G1-to-S transition (McGrath et al. 2006). At the same time, CtrA localizes to the same pole, where it is degraded by ClpXP (Fig. 1A; Ryan et al. 2004; McGrath et al. 2006). Two factors, which themselves sequester to the stalked cell pole, are responsible for the dynamic localization of the protease and its substrate. RcdA, a protein that interacts with CtrA in vivo, helps to localize CtrA to the pole (McGrath et al. 2006). Similarly, polarly localized CpdR tags ClpXP to the incipient stalked cell pole (Iniesta et al. 2006). CpdR is a member of the response regulator family, and its localization to the cell pole is negatively controlled by phosphorylation through the CckA-ChpT phosphorelay (Fig. 9A, below; Biondi et al. 2006; Iniesta et al. 2006).

These studies suggested that the cell cycle timing of CtrA degradation is intimately linked to its dynamic localization to the cell pole. But what are the molecular mechanisms that mediate RcdA and CtrA localization to this subcellular site, and how is this event temporally controlled during the cell cycle? Here we propose that the second messenger c-di-GMP critically contributes to temporal and spatial control of CtrA degradation during the $C$. crescentus cell cycle. C-di-GMP is a ubiquitous signaling molecule in bacteria, controlling the transition between a motile, single-cell and a sessile, surface-attached biofilm state in a wide range of organisms (Jenal and Malone 2006; Tamayo et al. 2007). Two opposing enzyme activities, diguanylate cyclase (DGC) and phosphodiesterase (PDE), control the cellular level of c-di-GMP. The DGC and PDE activities are contained within the highly conserved GGDEF and EAL domains, respectively (Paul et al. 2004; Christen et al. 2005). GGDEF and EAL domains are often associated with sensory input domains, and it is assumed that these regulatory proteins serve to directly couple environmental or internal stimuli to a specific cellular response through the synthesis or degradation of c-diGMP. DGCs are activated through dimerization of two GGDEF protomers (Paul et al. 2007; Wassmann et al. 2007). In addition, many DGCs are tightly controlled by product inhibition through the binding of c-di-GMP to an allosteric I-site, which is distinct from the catalytic active A-site (Chan et al. 2004; Christen et al. 2006).

We showed recently that $C$. crescentus pole morphogenesis during the swarmer-to-stalked cell transition is

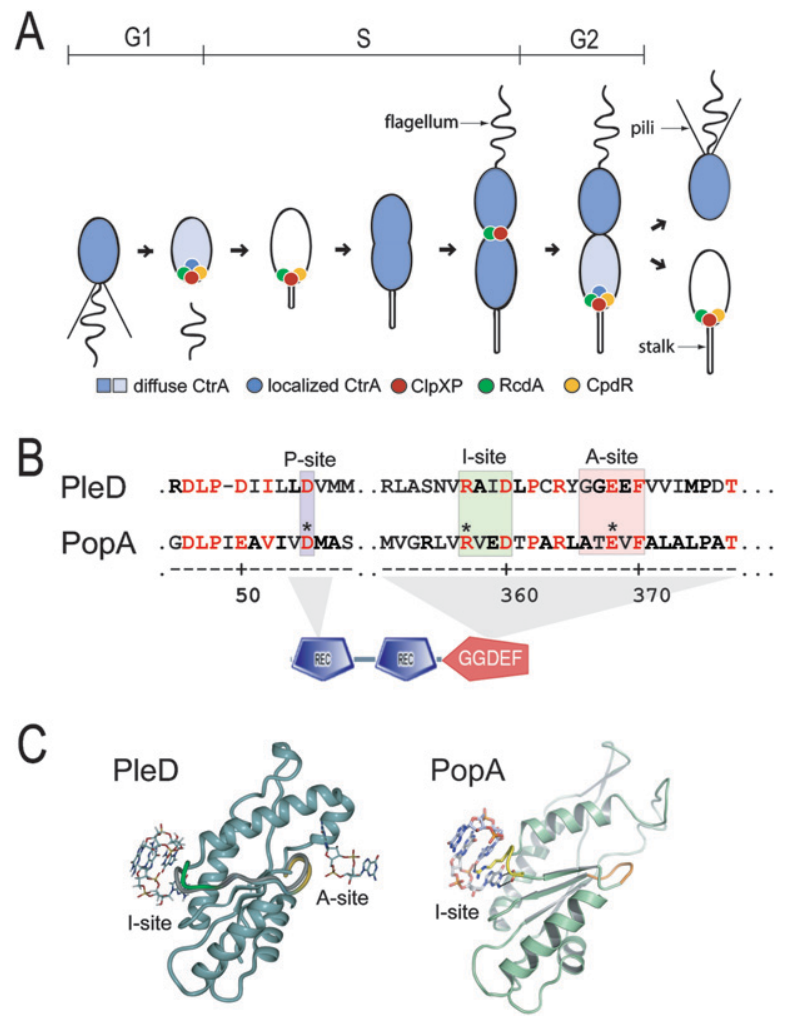

Figure 1. Dynamic protein localization and CtrA degradation during the cell cycle. (A) Schematic of CtrA, ClpXP, RcdA, and CpdR localization during the $C$. crescentus cell cycle. (B) Sequence alignment of the PleD and PopA. The amino acid sequence flanking the phosphoryl acceptor site (P-site), I-site, and A-site are shown with the conserved residues colored in red, the signature motifs boxed, and amino acids residues replaced in P-site, I-site, and A-site mutants marked with asterisks. $(C)$ Comparison of the 3D structure of the GGDEF domains of PleD (as determined by X-ray crystallography) (Chan et al. 2004) and modeled PopA. A-sites and I-sites are marked, and the position of a dimer of c-di-GMP bound to the I-site is indicated.

controlled by the DGC PleD (Aldridge et al. 2003; Paul et al. 2004; Levi and Jenal 2006). During development, $\mathrm{PleD}$ is activated by phosphorylation and in response is sequestered to the differentiating cell pole, where it is believed to activate its downstream targets (Paul et al. 2004, 2007). Here we analyze the role of the PleD paralog PopA in C. crescentus development and cell cycle progression. Similar to PleD, PopA is sequestered to the old cell pole. PopA localization does not require phosphorylation but depends on c-di-GMP binding to the conserved I-site of its GGDEF output domain. We demonstrate that PopA directly interacts with RcdA and helps to recruit both RcdA and CtrA to the cell pole. Based on our data, we postulate that c-di-GMP binding dynamically sequesters PopA to the old cell pole during the G1-to-S transition, where it helps to recruit the machinery responsible for CtrA degradation. This establishes the GGDEF domain as a bona fide c-di-GMP effector module and discovers a novel role for c-di-GMP in interfering with the central machinery driving cell proliferation. 


\section{Results}

PopA is a structural homolog of the PleD diguanylate cyclase

In the course of the functional characterization of $C$. crescentus proteins involved in c-di-GMP turnover, we analyzed ORF CC1842. This gene codes for a response regulator with two receiver domains and a GGDEF output domain (Fig. 1B). Because of its homology with the diguanylate cyclase PleD, CC1842 was renamed popA (paralog of pleD). Sequence comparison of PleD and PopA revealed that the phosphoryl acceptor site (Asp55; P-site) of the first receiver domain and the I-site motif (RVED) of the GGDEF domain were conserved, while the catalytic A-site motif was degenerate (Fig. 1B). Based on this homology (23\% identity) (Supplemental Fig. S1), the overall fold of the receiver domains and the GGDEF domain can be expected to be conserved. A three-dimensional (3D) model of the PopA structure was built using the crystal structure of PleD (Chan et al. 2004) as template. Despite the low sequence conservation, the modeled PopA structure was similar to PleD (Fig. 1C), suggesting that the overall fold of the receiver domains and the GGDEF domain is conserved. In particular, the arrangement of amino acids contributing to the high-affinity binding site for c-di-GMP (I-site) is identical (Fig. 1C).

\section{PopA is required for cell cycle-dependent degradation} of $\operatorname{Ctr} A$

A chromosomal popA in-frame deletion mutant was generated and analyzed for several morphological and cell cycle-associated markers. The mutant strain showed a significantly reduced motility on semisolid agar plates compared with wild type (data not shown), suggesting a specific defect in motor function or in timing of motility during the cell cycle. Moreover, the $\Delta p o p A$ mutant failed to degrade the cell cycle regulator CtrA upon entry into $S$ phase (Fig. 2A). Cell cycle-dependent degradation of the chemoreceptor McpA, another ClpX substrate (Tsai and Alley 2001), was unaffected (Fig. 2A). To test which PopA motif is required for CtrA degradation, we generated mutations in the conserved P-site (D55N) and I-site (R357G), and in the degenerate A-site (E368Q) (Fig. 1B). Analysis of CtrA turnover in the respective mutant strains revealed that CtrA was degraded normally in the P-site and A-site mutants but stabilized in the I-site mutant (Fig. 2A).

To confirm that PopA interferes with CtrA stability, wild-type and $\triangle p o p A$ mutant strains were engineered that expressed the YFP-CtrARD +15 allele from the xylose-inducible promoter $\mathrm{P}_{\mathrm{xylX}}$. The YFP-CtrARD +15 fusion protein is a fluorescent CtrA derivative, which contains the minimal requirements for polar sequestration and cell cycle-regulated proteolysis (Ryan et al. 2002). Cells grown in the presence of xylose were synchronized and released into fresh minimal medium lacking xylose. In wild-type cells, both full-length CtrA and YFP-CtrARD +15 were degraded normally during the G1-to-S transition (Fig. 2B; Supplemental Fig. S2A). However, synthesis of the fusion protein did not resume after cells had
A
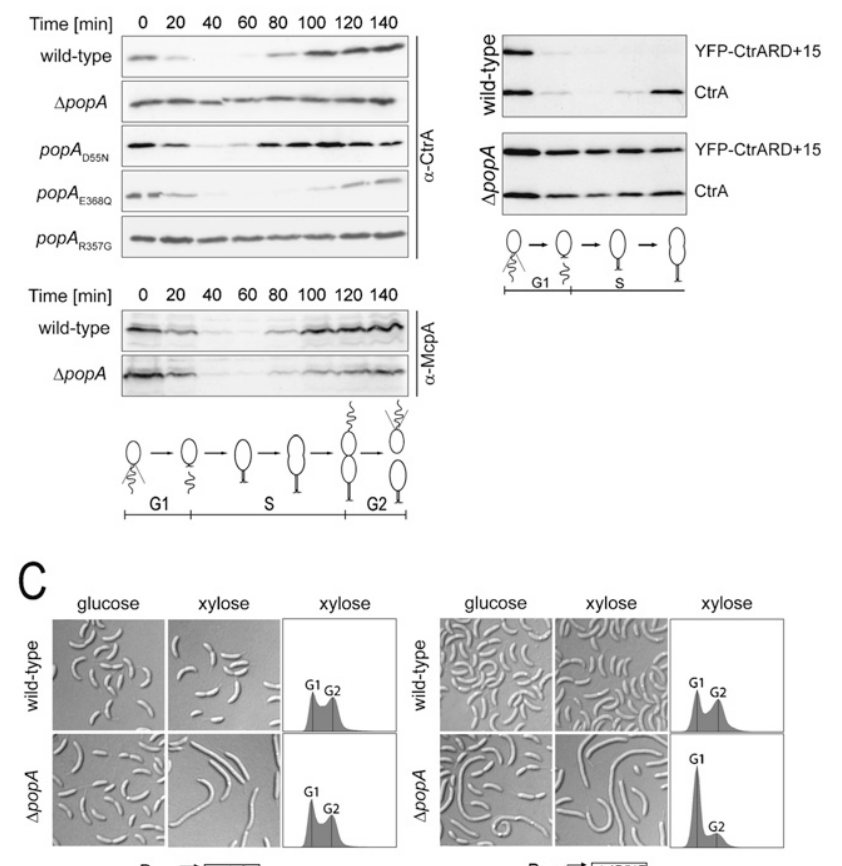

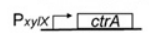

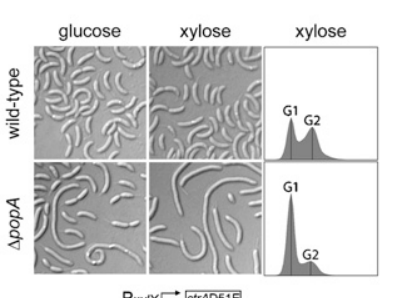

Figure 2. Cell cycle-dependent degradation of CtrA requires PopA. (A) Immunoblots of synchronized cultures of C. crescentus wild-type and popA mutant strains. Immunoblots were stained with anti-CtrA or anti-McpA antibodies as indicated. $(B)$ Synchronized swarmer cells of wild type (top panel) and popA mutant (bottom panel) expressing yfp-ctrARD + 15 from the xylose-inducible promoter $\mathrm{P}_{\mathrm{xyl}}$ were released into $\mathrm{M} 2 \mathrm{G}$ minimal glucose medium and monitored throughout the cell cycle using anti-CtrA antibodies. The YFP-CtrARD + 15 fusion protein and wild-type CtrA are labeled. (C) Morphology and replication status of $C$. crescentus wild type and $\triangle p o p A$ mutant expressing ctrA (left panels, strains UJ3966 and UJ3969) or ctrA $A_{D 51 E}$ (right panels, strains UJ3967 and UJ3970) from the xylose-inducible promoter $\mathrm{P}_{\mathrm{xyl}}$. Cells were harvested under inducing (xylose) or non-inducing (glucose) conditions and analyzed by light microscopy and by flow cytometry.

entered $S$ phase, confirming that the $y f p$-ctrARD +15 allele was not expressed under these conditions (Fig. 2B). In the $\Delta$ popA mutant, the YFP-CtrARD +15 fusion protein was stabilized (Fig. 2B; Supplemental Fig. S2A). Similarly, the overall half-life of CtrA in nonsynchronous populations was significantly increased in a popA mutant (Supplemental Fig. S2B).

Mutants that are unable to remove active CtrA during the cell cycle show a distinct G1 arrest and cell division block (Domian et al. 1997). To test if popA mutants display a similar cell cycle arrest, plasmid-borne copies of $\operatorname{ctr} A$ wild type and $\operatorname{ctr} A D 51 E$, which codes for a constitutive active form of the regulator (Domian et al. 1997), were expressed from the xylose-inducible promoter. The expression of $\operatorname{ctr} A$ or $\operatorname{ctrAD51E}$ had no effect on $C$. crescentus wild-type cells. In contrast, the pop $A$ mutant showed a weak but noticeable cell division and replication defect upon expression of wild-type $\operatorname{ctr} A$ and a strong 
G1 arrest upon expression of $\operatorname{ctrAD51E}$ (Fig. 2C). This phenotype was comparable with the G1 arrest observed in $r c d A$ or $c p d R$ mutants expressing the $c t r A D 51 E$ allele or in cells expressing a constitutive active and stabilized form of CtrA (CtrAD51E $\Delta 3 \Omega$ ) (Supplemental Fig. S3).

To conclude, these data suggest that PopA is required for the cell cycle-dependent degradation of the CtrA master regulator and to promote entry of $C$. crescentus cells into $S$ phase.

\section{PopA is required for CtrA and RcdA sequestration to the cell pole}

To analyze at which level PopA interferes with CtrA degradation, we first examined the cellular position of the YFP-CtrARD + 15 fusion protein in the $\Delta p o p A$ mutant. Whereas YFP-CtrARD + 15 transiently localizes to the cell poles in C. crescentus wild-type cells, CtrA foci were not present in stalked or predivisional cells of the $\Delta p o p A$ mutant (Fig. 3A). Instead, an intense diffuse fluorescence was observed in all $\Delta p o p A$ mutant cells, indicative of a stabilized CtrA fusion protein. These results indicated that PopA is involved in polar sequestration of CtrA.
Next we asked if PopA was required for the localization of RcdA. As shown in Figure 3B, an RcdA-eGFP fusion localized to the old cell pole in wild-type cells but failed to sequester to the pole in the $\Delta p o p A$ or in the $p o p A_{R 357 G}$ I-site mutant. Based on these results, we propose that PopA is positioned upstream of RcdA in the signal transduction cascade leading to cell cycle-dependent degradation of CtrA and that PopA directs CtrA to the cell pole via the localization of RcdA.

Because RcdA interacts with CtrA and ClpX in vivo (McGrath et al. 2006), we examined if PopA also interacts with any of these factors. For this, we used the bacterial adenylate cyclase two-hybrid (BACTH) system, which is based on the interaction-mediated reconstruction of a cyclic AMP (cAMP) signaling cascade (Karimova et al. 1998). Fusions between PopA, RcdA, CpdR, CtrA, ClpX, or ClpP and two complementary fragments, T25 and T18, that constitute the catalytic domain of Bordetella pertussis adenylate cyclase were generated in all possible combinations and assayed for cAMP production on maltose MacConkey agar plates. Strong signals indicating interaction were obtained for the following protein pairs:
A

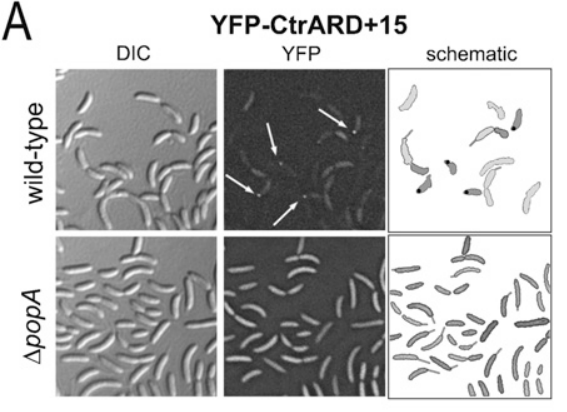

B

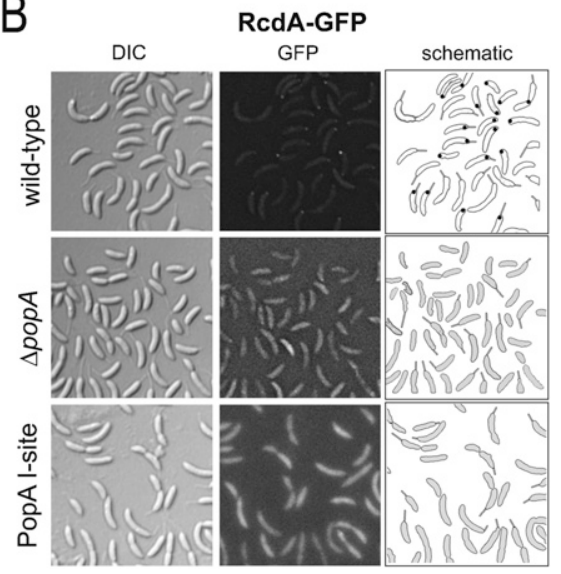

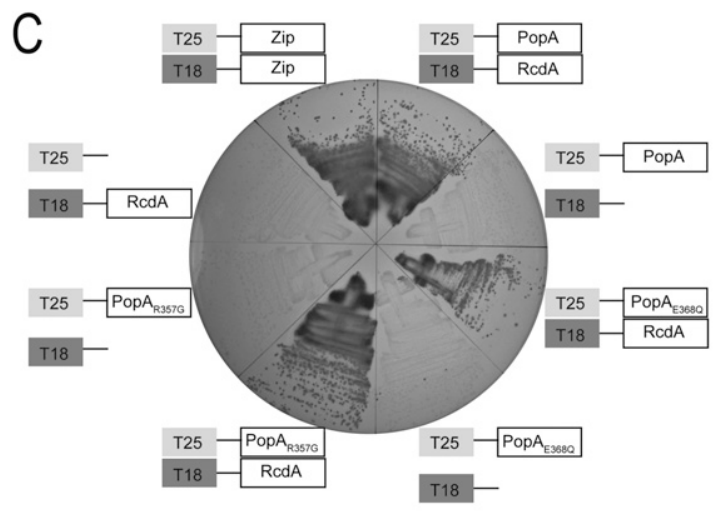
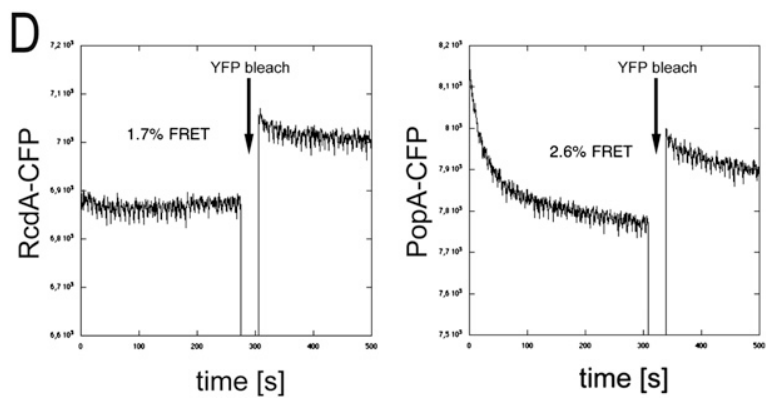

Figure 3. PopA mediates polar localization of RcdA and CtrA. (A) Wild-type and $\triangle p o p A$ mutant cells expressing yfp-ctrARD+15 were analyzed by DIC and fluorescence microscopy. Polar foci of Yfp-CtrARD + 15 are marked by arrows and shown schematically in the right panel. (B) Wild-type, $\Delta p o p A$, and $p o p A_{R 357 G}$ (I-site) mutant cells expressing $r c d A$-yfp were analyzed by DIC and fluorescence microscopy. (C) PopA directly interacts with RcdA. The red color on McConkey agar-base maltose plates is an indicator for proteinprotein interaction. PopA and RcdA fusions to the adenylate cyclase fragments T18 and T25 are indicated. Zip indicates a positive control. (D) In vivo FRET analysis of PopA and RcdA. C. crescentus cells expressing rcdA-ecfp and popA-eyfp (left panel) or popA-ecfp and $r c d A$-eyfp (right panel) were analyzed. The intensity of the CFP channel was recorded before and after YFP-specific bleaching. The increase of signal intensity of the CFP channel after specific bleaching of the YFP channel is a measure of the FRET efficiency (\%). 
ClpX/ClpX, ClpP/ClpP, and ClpX/CpdR (Supplemental Fig. S4B). This is in agreement with earlier results demonstrating ClpX-CpdR interaction by coimmunoprecipitation (Iniesta et al. 2006) or with ClpX and ClpP forming oligomeric complexes (Wang et al. 1998; Kim and Kim 2003). In addition, we obtained a strong positive signal for the interaction between PopA and RcdA (Fig. 3C). Interaction with RcdA as measured by the twohybrid system did not require an intact PopA I-site or A-site (Fig. 3C). Weak but reproducible interaction signals were obtained for PopA/PopA and for CpdR/RcdA. No interactions were detected between PopA and CtrA and between PopA and CpdR (Supplemental Fig. S4B). This result adds PopA to the protein-protein interaction map of the CtrA degradation machinery as outlined in Supplemental Figure S4A.

To measure the interaction between PopA and RcdA in live Caulobacter cells, we used fluorescence resonance energy transfer (FRET), which relies on the distance-dependent transfer of energy from an excited donor fluorophore to an acceptor fluorophore (Miyawaki and Tsien 2000; Selvin 2000; Sourjik and Berg 2002). We engineered two $C$. crescentus strains containing plasmid-borne copies of either popA-cfp and $r c d A-y f p$ or popA-yfp and $r c d A-c f p$. Both strains were used to perform FRET measurements by quantifying the difference of the CFP donor fluorescence before and after specific bleaching of the YFP acceptor (see the Materials and Methods) (Sourjik and Berg 2002). For both strains, clear differences in CFP fluorescence intensity were measured, indicative of a direct interaction between the two partners (Fig. 3D). To conclude, we propose that PopA directly interacts with RcdA and by mediating RcdA localization to the old cell pole directs CtrA to this subcellular site during the G1-to-S transition.

PopA localizes to the new and old cell pole in an RcdA- and ClpX-independent manner

The observation that PopA directly interacts with RcdA and directs this small protein to the C. crescentus cell pole prompted us to test if PopA itself is sequestered to the cell pole, where it could act as a pole specificity factor for RcdA. To observe the intracellular position of PopA, we constructed a popA-egfp fusion expressed from its own promoter on a low-copy-number plasmid. The PopAeGFP fusion protein was fully functional as $\Delta p o p A$ mutant cells carrying a plasmid-borne popA-egfp allele showed a wild-type motility and CtrA turnover phenotype (data not shown). As shown in Figure 4A and Supplemental Figure S5, PopA-eGFP localizes to the cell poles throughout the cell cycle. A single focus appeared at one pole of the incipient swarmer cell. Because predivisional cells show a focus at both the stalked and the flagellated pole, we presume that the focus observed in newborn swarmer cells occupies the old flagellated pole. During the G1-to-S transition PopA-eGFP dynamically positions to the new cell pole, resulting in a bipolar distribution pattern in stalked and predivisional cells (Fig. 4A; Supplemental Fig. S5; Supplemental Movie S13). It is important to note that in stalked and predivi-

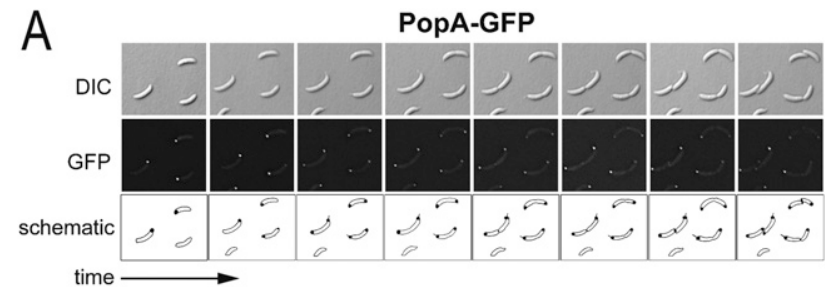

B

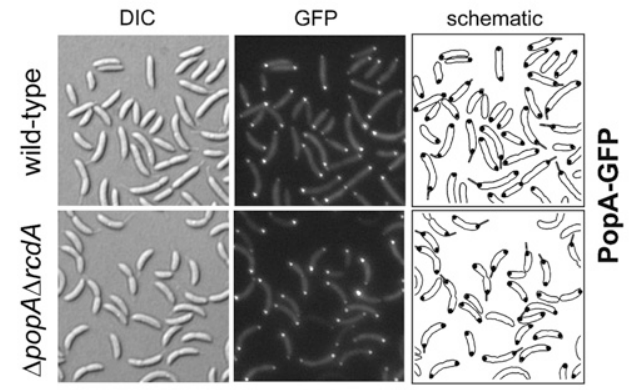

C

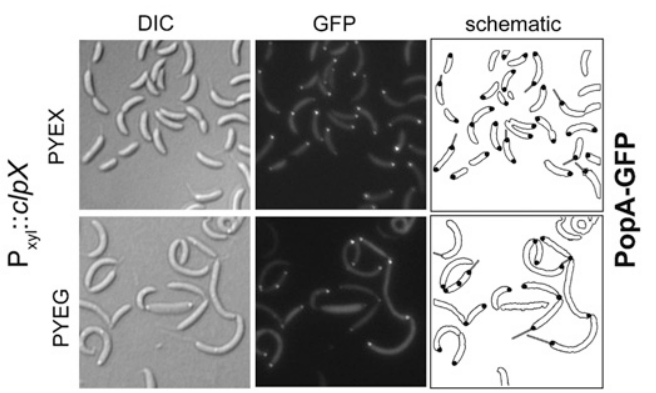

Figure 4. PopA localizes to the old and new cell poles. $(A)$ Time-lapse experiment with synchronized $C$. crescentus wildtype cells expressing popA-egfp. The first sample corresponds to the 0 -min time point, and samples were analyzed every $20 \mathrm{~min}$. $(B)$ RcdA is dispensable for PopA localization to the cell poles. $C$. crescentus wild-type and $\Delta r c d A$ mutant cells expressing popAegfp were analyzed by DIC and fluorescence microscopy. $(C)$ $\mathrm{ClpX}$ is dispensable for PopA localization to the cell poles. Mixed cultures of the $C$. crescentus conditional clpX mutant strain UJ271 expressing popA-egfp were analyzed by DIC and fluorescence microscopy under permissive (PYEX) and restrictive conditions (PYEG). Cultures were grown in the absence of xylose for $6 \mathrm{~h}$, resulting in complete depletion of ClpX (data not shown).

sional cells, the fluorescence intensity is different at the two cell poles, with stronger foci always marking the old stalked cell pole. After cell division, PopA-eGFP asymmetrically positions to the new pole of the daughter stalked cell, while the new pole inherited by the swarmer progeny remains unoccupied during most of the G1 phase (Fig. 4A; Supplemental Fig. S5; Supplemental Movie S13).

Because polar positioning of RcdA requires both ClpX (McGrath et al. 2006) and PopA (Fig. 3B), we wanted to test if PopA localization also requires one of these factors. As shown in Figure 4, B and C, PopA-eGFP localization was unaltered in mutants either lacking RcdA or being depleted for ClpX. Based on this, we propose that PopA is at the top of the recruitment and degradation hierarchy for CtrA and that this factor is primarily required for the 
spatiotemporal behavior associated with CtrA degradation during the cell cycle.

PopA localization to the old cell pole is c-di-GMP-dependent

If PopA is required for the spatiotemporal control of RcdA and CtrA upon S-phase entry, one would expect that the dynamic sequestration of the three proteins to the cell pole more or less coincides. However, PopA localizes to the new pole long before RcdA and CtrA are sequestered to the same subcellular site. It is possible that PopA works in conjunction with (an) additional factor(s) responsible for temporal control of CtrA degradation. Alternatively, the cellular dynamics and specificities of PopA might be more complex and thus not apparent from analyzing its overall distribution in wild-type cells. To test this possibility, we analyzed the molecular basis of PopA sequestration to the cell poles. The observation that in the pop $A_{R 357 G}$ I-site mutant, RcdA failed to mediate CtrA degradation even though PopA $\mathrm{R}_{\mathrm{R} 37 \mathrm{G}}$ was still able to interact with RcdA suggested that an intact Isite might be required for polar localization of PopA rather than for the subsequent recruitment of RcdA. To test this hypothesis, low-copy-number plasmids containing the mutant alleles pop $A_{D 55 N^{-}} \operatorname{egfp}\left(\mathrm{P}\right.$-site), $p o p A_{R 357 G^{-}}$ egfp (I-site), and pop $A_{E 368 Q^{-}}$egfp (A-site) were constructed and introduced into the $C$. crescentus wild-type and $\Delta p o p A$ mutant strains. In accordance with their wildtype-like CtrA degradation behavior, both PopA P-site and A-site mutants showed a localization pattern indistinguishable from PopA wild type (Fig. 5; Supplemental Fig. S6A). In contrast, PopA $\mathrm{R}_{357 \mathrm{G}}-\mathrm{eGFP}$ showed a characteristic unipolar localization pattern (Fig. 5; Supplemental Fig. S6B). Noticeably, the PopA I-site mutant failed to localize to the stalked cell pole but was still able to sequester to the opposite pole of the cell. As a result of

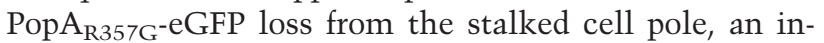
creased diffuse fluorescence was observed in all cells. Because of the asymmetric positioning of PopA $\mathrm{R}_{\text {357G- }}$ eGFP in predivisional cells, newborn swarmer cells inherited a fluorescent focus at the old flagellated pole. During the G1-to-S transition PopA $\mathrm{R}_{\mathrm{R} 37 \mathrm{G}}$-eGFP was rapidly lost from the old pole after 20 to $40 \mathrm{~min}$ and appeared at the opposite new pole after 40 to $60 \mathrm{~min}$ (Supplemental Fig. S6B).

The failure of PopA $\mathrm{R}_{\mathrm{R} 37 \mathrm{G}}$ to sequester to the ClpXPoccupied old pole and to promote CtrA degradation suggested a specific role for I-site-mediated c-di-GMP binding in PopA dynamics and CtrA recruitment. In agreement with this, the C-terminal GGDEF domain is required for polar localization of PopA (Supplemental Fig. S7; Supplemental Table S2). To identify a potential diguanylate cyclase involved in PopA sequestration, we analyzed single mutants lacking each of the 12 genes for GGDEF and EAL domain proteins encoded in the $C$. crescentus genome. None of the mutants showed altered localization of PopA (data not shown) arguing that multiple c-di-GMP signaling components control PopA behavior redundantly. To provide direct evidence for the role of c-di-GMP in the PopA-CtrA pathway, we expressed the

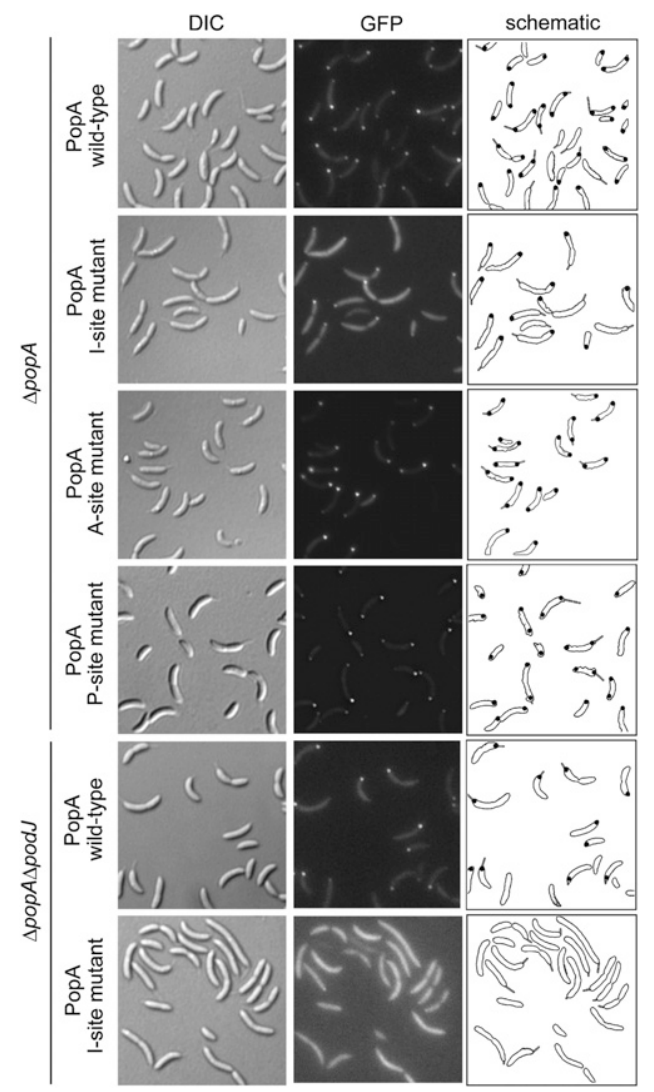

Figure 5. Distinct mechanisms mediate PopA localization to the new and old pole. C. crescentus wild type and podJ mutant expressing GFP fusion proteins to PopA wild type and the following PopA mutants were analyzed by DIC and fluorescence microscopy: PopA $A_{\mathrm{D} 55 \mathrm{~N}}$ (P-site mutant), PopA $\mathrm{A}_{\mathrm{E} 368 \mathrm{Q}}$ (A-site mu$\operatorname{tant}), \operatorname{Pop}_{\mathrm{R} 357 \mathrm{G}}$ (I-site mutant). Polar localization is indicated schematically in the panels on the right.

gene encoding a c-di-GMP-specific phosphodiesterase from Pseudomonas aeruginosa, PA5295 (Kulasakara et al. 2006), from the inducible vanillate promoter (Thanbichler et al. 2007) in C. crescentus cells harboring a PopA-eGFP fusion. In agreement with its proposed role as PDE, expression of PA5295 in C. crescentus reduced the cellular levels of c-di-GMP below the detection limit (Supplemental Fig. S8A). Induction of PA5295, but not of the active site mutant $P A 5295_{E 328 A}$, rapidly blocked polar localization of PopA-eGFP (Fig. 6A) as well as CtrA (Supplemental Fig. S8B) and completely stabilized CtrA during the G1-to-S transition (Fig. 6B). In summary, we propose that PopA is able to respond to fluctuating levels of c-di-GMP by I-site-mediated binding, that this mediates sequestration of PopA to the stalked cell pole, and that PopA dynamics contributes to the timing of CtrA turnover during the $C$. crescentus cell cycle.

The unipolar localization pattern of PopA $\mathrm{R}_{\mathrm{R} 57 \mathrm{G}}$-eGfp suggested that an additional mechanism is required to specifically sequester PopA to the new cell pole. Because the motility defect of the $\Delta p o p A$ mutant is similar to the phenotype described for a podJ mutant (Wang et al. 1993) 
A

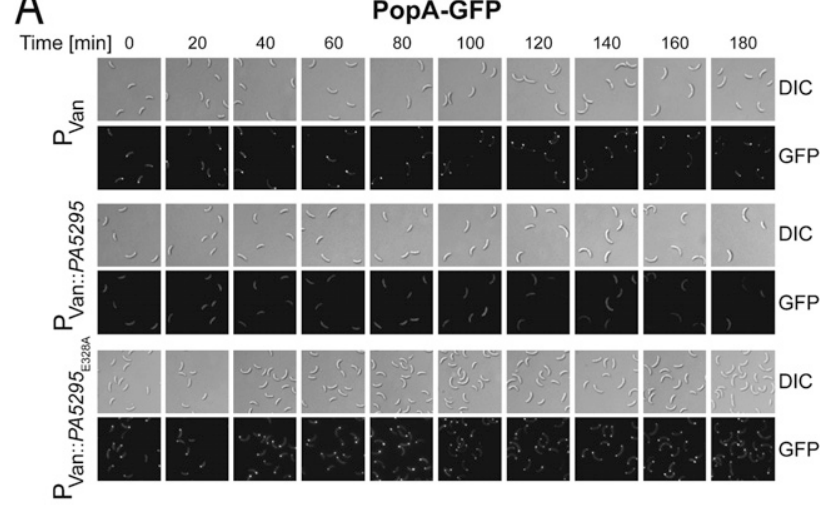

B

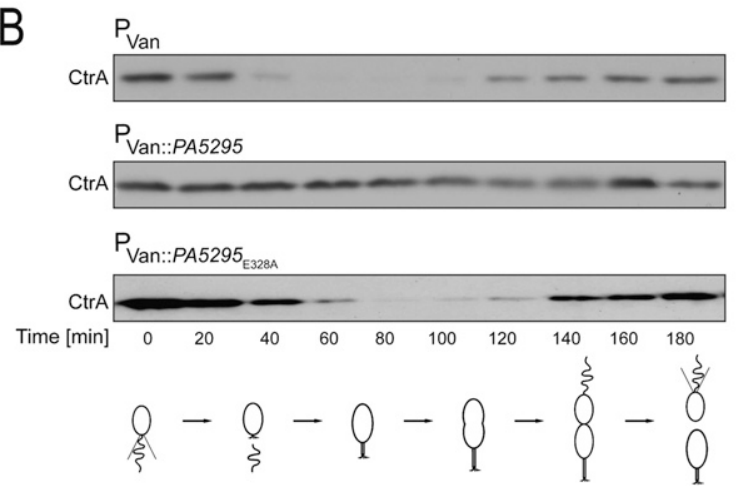

Figure 6. Expression of a heterologous phosphodiesterase interferes with PopA localization and CtrA degradation. Cell cycledependent PopA-GFP localization $(A)$ and CtrA degradation $(B)$ in cells of $C$. crescentus $\mathrm{CB} 15$ harboring a vanillate induced copy of PA5295 from P. aeruginosa (middle panels) or an active site mutant, PA5295 E328A (bottom panels). Control assays with cells harboring the empty vector (Pvan) are shown in the top panel.

and because PodJ functions as a swarmer pole-specific protein localization factor (Viollier et al. 2002), we tested if PopA localization to the new cell pole was dependent on PodJ. As shown in Figure 5 and Supplemental Table S3, PopA-eGFP primarily localized to the stalked pole in a $\Delta$ podI mutant. When the distribution of the I-site mutant Pop $\mathrm{A}_{\mathrm{R} 357 \mathrm{G}}$-eGfp was analyzed in the $\Delta$ podJ mutant, the polar foci were replaced by a strong diffuse cytoplasmic fluorescence (Fig. 5; Supplemental Table S3), arguing that both polar addresses had been concealed. Based on these results, we conclude that PopA has two independent and function-specific addresses at the new and old cell poles, respectively.

PopA lacks DGC activity but binds c-di-GMP specifically and with high affinity

Because the I-site was originally identified as an allosteric binding site that regulates diguanylate cyclase activity of GGDEF domains (Chan et al. 2004; Christen et al. 2006; Wassmann et al. 2007), we first analyzed if PopA, despite its degenerate A-site, shows enzymatic activity. A hexahistidine-tagged version of PopA was purified and used for DGC in vitro activity assays (Paul et al. 2004; Christen et al. 2006). PopA failed to show DGC activity even at the highest protein concentrations used (data not shown). This is in agreement with the observation that most amino acid changes in the highly conserved GGDEF signature motif abolished enzyme activity of an active DGC (Malone et al. 2006).

Next we used a UV cross-link assay (Christen et al. 2006) with radiolabeled c-di-GMP to assay ligand binding of PopA wild type, PopA $\mathrm{E}_{\mathrm{E} 368 \mathrm{Q}}$ (A-site mutant), and PopA $_{\mathrm{D} 357 \mathrm{G}}$ (I-site mutant). Whereas both PopA wild type and $\mathrm{PopA}_{\mathrm{E} 368 \mathrm{Q}}$ readily bound c-di-GMP, ligand binding was abolished in the I-site mutant protein PopA $_{\mathrm{D} 357 \mathrm{G}}$ (Fig. 7A). Binding of radiolabeled ligand was then assayed in the presence of increasing concentrations of nonlabeled c-di-GMP to determine binding affinity. As shown in Figure $7 \mathrm{~B}$, PopA binds c-di-GMP with a $K_{\mathrm{d}}$ of $\sim 2 \mu \mathrm{M}$. Other nucleotides like GTP or GDP were not able to chase radiolabeled c-di-GMP, suggesting that binding of c-di-GMP to PopA is highly specific (data not shown). Together this implied that PopA specifically binds c-diGMP via its conserved I-site motif and that it uses this site to modulate its own activity and dynamic behavior in response to fluctuating levels of c-di-GMP.

CpdR and PopA pathways converge, leading to cell cycle-dependent degradation of CtrA

To analyze if PopA, in addition to its role in RcdA and CtrA sequestration, is also involved in the recruitment of the ClpXP protease to the cell pole, we assayed ClpX localization during the $C$. crescentus cell cycle. In C. crescentus wild type, ClpX localizes to the old cell pole during the G1-to-S transition, coinciding with CtrA degradation (Supplemental Figs. S9, S10; note that cell
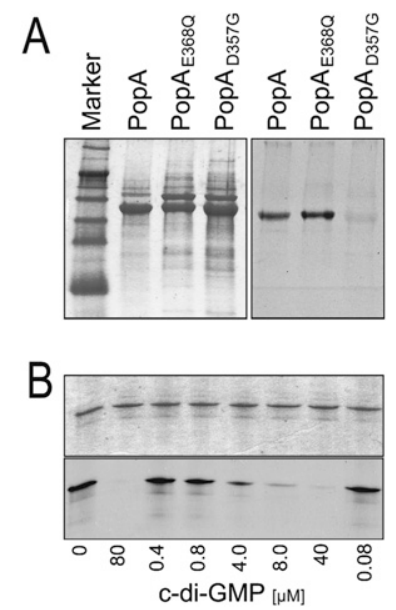

Figure 7. PopA binds c-di-GMP at the conserved I-site of the GGDEF domain. (A) UV cross-link experiment of purified hexahistidine-tagged PopA with $\left[{ }^{33} \mathrm{P}\right]$-labeled c-di-GMP. The following proteins were used: PopA, PopA $\mathrm{E}_{\mathrm{E} 368 \mathrm{D}}$ (A-site mutant), PopA $_{\mathrm{R} 357 \mathrm{G}}$ (I-site mutant). The Coomassie blue-stained gel (left) and the autoradiograph (right) are shown. (B) UV cross-linking of purified PopA with $\left[{ }^{33} \mathrm{P}\right]$-labeled c-di-GMP and increasing concentrations of nonlabeled c-di-GMP $(0-80 \mu \mathrm{M})$. Coomassie bluestained gel (top panel) and autoradiograph (bottom panel) are shown. 
cycle timing in Supplemental Figs. S9 and S10 is identical to the experiments shown in Fig. 2A; Iniesta et al. 2006). In the $\Delta p o p A$ mutant ClpX, localization was impaired (Supplemental Figs. S9, S10). Reduction of ClpX localization in popA mutants paralleled with a similar reduction in the localization of the ClpXP targeting factor CpdR (Supplemental Figs. S9, S12). Thus, we conclude that PopA in addition to being required for substrate sequestration is also needed for efficient recruitment of CpdR and ClpXP to, or retention at, the old cell pole. In contrast, PopA localization to the stalked pole is not affected in a $c p d R$ mutant (Supplemental Fig. S11).

The observation that distinct targeting factors and mechanisms are involved in ClpXP and CtrA localization raised the questions if two independent polar recruitment pathways ultimately converge to determine temporal control of CtrA degradation during the cell cycle. To test this, we analyzed CtrA stability in popA mutants that also carried a functional $c p d R_{D 51 A}$-YFP mutant allele. The $\mathrm{CpdR}_{\mathrm{D} 51 \mathrm{~A}}$ mutant localizes to the cell pole more effectively as compared with wild-type CpdR (Fig. 8A) and causes increased CtrA turnover by more efficient polar recruitment of the ClpXP protease (Iniesta et al. 2006). In agreement with this, we find severely reduced levels of CtrA in cells expressing $c p d R_{D 51 A}$ (Fig. 8B). This was due to increased degradation of CtrA, as normal CtrA
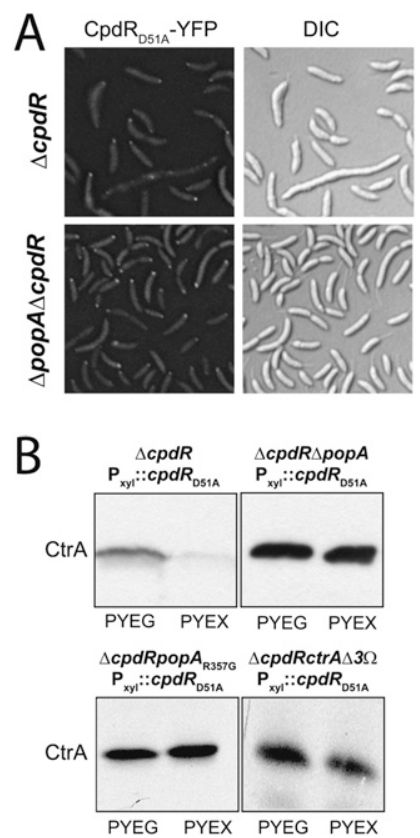

Figure 8. CpdR and PopA constitute two converging pathways leading to cell cycle-dependent degradation of CtrA. (A) Cultures of $C$. crescentus $\Delta c p d R$ single and $\Delta c p d R \Delta p o p A$ double mutants expressing $c p d R_{D 51 A}-y f p$ under the control of the xylose-inducible promoter $\mathrm{P}_{\mathrm{xyl}}$ were grown in the presence of xylose and analyzed microscopically. (B) Cultures of different $C$. crescentus $\Delta c p d R$ and $\Delta p o p A$ mutants expressing $c p d R_{D 51 A^{-}} y f p$ under the control of the xylose-inducible promoter $\mathrm{P}_{\mathrm{xyl}}$ were grown in the presence (PYEX) or absence of xylose (PYEG) and analyzed by immunoblots using anti-CtrA antibodies. levels were restored in cells coexpressing the stable variant $\operatorname{CtrA} \Delta 3 \Omega$ (Fig. 8B). Surprisingly, while cells carrying the $c p d R_{D 51 A}$ allele showed a severe filamentation and bulging phenotype in a pop $A$ wild-type background, they had a normal morphology in a $\Delta p o p A$ mutant (Fig. $8 \mathrm{~A})$. This effect was not due to reduced localization of $\mathrm{CpdR}_{\mathrm{D} 51 \mathrm{~A}}$-YFP in the $\Delta p o p A$ mutant (Fig. 8A). Strikingly, in the $\Delta$ popA-null mutant or the popA I-site mutant (R357G), normal CtrA levels and cell morphology were restored even when the expression of the $c p d R_{D 51 A}$ allele was induced (Fig. 8B).

In summary, these data provide evidence that the CpdR and PopA localization pathways converge and that CtrA degradation is mediated through the concomitant dynamic localization of these factors to the old cell pole during the G1-to-S transition.

\section{Discussion}

C. crescentus G1-to-S cell cycle progression is mediated by the degradation of the master regulator CtrA. To understand how this event is regulated and to elucidate the general control mechanisms operating during the bacterial cell cycle, we examined the spatiotemporal behavior of CtrA degradation. CtrA localization to the cell pole is mediated by RcdA, a small, stalked polespecific protein that interacts with CtrA and ClpXP in vivo (McGrath et al. 2006). We show here that RcdA also interacts with PopA, a GGDEF domain protein required for CtrA degradation and RcdA localization. The strong positive signals observed with the bacterial two-hybrid system and by FRET analysis indicated that PopA directly interacts with RcdA. Based on this and on epistasis experiments that positioned PopA upstream of RcdA, we propose that PopA directs CtrA to the cell pole via its interaction with RcdA, which in turn might play an intermediary role between CtrA and PopA (Fig. 9). In vitro experiments have argued against the idea that RcdA enhances CtrA degradation by tethering the substrate to its protease (Chien et al. 2007). However, RcdA could contribute to the timing of CtrA polar localization and/or confer substrate specificity to the cellular machinery that recruits proteins destined for degradation to the ClpXPtagged cell pole.

If PopA is at the top of the cascade that determines CtrA sequestration and degradation, what controls its activity during the cell cycle? We found that PopA localizes to the new and the old cell poles at distinct times of the cell cycle. Remarkably, successive localization of PopA to these two subcellular sites relies on distinct mechanisms, which apparently serve different cellular functions. PopA localization to the incipient swarmer pole requires PodJ, a cell polarity determinant that also recruits the $\mathrm{PleC}$ histidine kinase/phosphatase and components of the pili assembly machinery to this site (Viollier et al. 2002; Hinz et al. 2003; Lawler et al. 2006). Whereas PodJ is not required for CtrA degradation and is cleared from the cell pole before cells enter $\mathrm{S}$ phase (Chen et al. 2006), PopA persists at this subcellular site, where it facilitates CtrA degradation. Several lines 

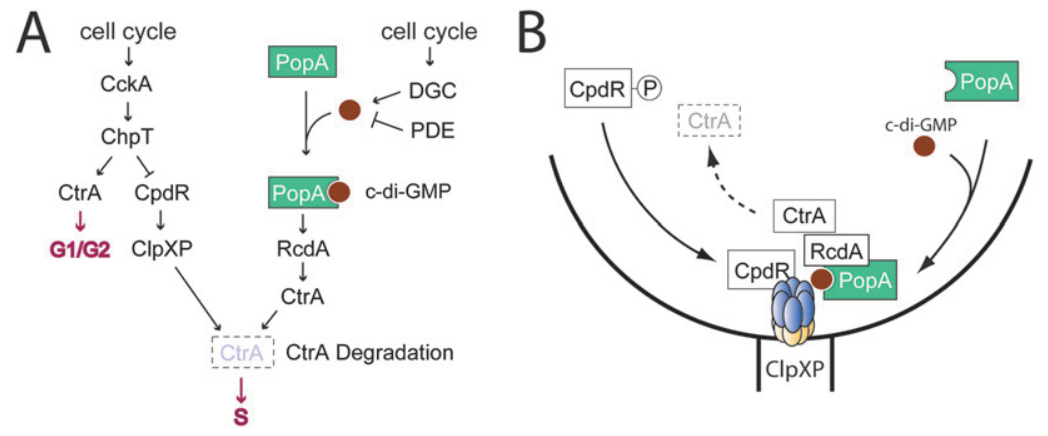

Figure 9. Model for the role of PopA in cell cycledependent degradation of CtrA. (A) Converging pathways involved in polar sequestration of ClpXP and its substrate CtrA. The CckA-ChpT phosphorelay inversely regulates CtrA activity and stability through the phosphorylation of CtrA and CpdR. The model predicts that cell cycle-dependent localization of PopA to the stalked cell pole involves the timed synthesis and/or hydrolysis of c-di-GMP by as-yet-unidentified DGCs or PDEs. (B) Upon binding of c-di-GMP, PopA sequesters to the cell pole, where it recruits RcdA and CtrA. of evidence suggest a direct involvement of c-di-GMP in PopA recruitment to the incipient stalked pole. This process requires an intact I-site, but not the P-site or A-site. Both modeling and biochemical experiments indicated that the conserved I-site of PopA is a high-affinity binding site for c-di-GMP. The affinity of PopA for c-diGMP is similar to the allosteric I-sites of two enzymatically active DGCs of C. crescentus (Chan et al. 2004; Christen et al. 2006; Wassmann et al. 2007), suggesting that PopA binds c-di-GMP within a physiologically relevant concentration range (Christen et al. 2006). Specific mutations in this pocket disrupt c-di-GMP binding and interfere with PopA localization to the stalked pole. Also, PopA lacks the highly conserved GGDEF signature motif of the DGC active site (Malone et al. 2006; Wassmann et al. 2007), and biochemistry experiments failed to detect PopA DGC activity. This suggests that the GGDEF domain of PopA has adopted a novel role as a c-di-GMP-binding device. The observation that an intact I-site is required for PopA sequestration to the stalked cell pole, but not for protein-protein interaction with RcdA, argues that c-di-GMP binding facilitates the timing of PopA sequestration to the old cell pole, rather than its interaction with downstream components. Finally, upon expression of a heterologous phosphodiesterase, we observed rapid and specific interference with PopA localization to the old cell pole and with CtrA degradation. This strongly suggests that depletion of the cellular c-di-GMP pool inactivates the PopA pathway by interfering with its sequestration to the cell pole.

We showed recently that c-di-GMP levels peak during the G1-to-S transition and that the PleD diguanylate cyclase is mainly responsible for this fluctuation (Paul et al. 2008). However, PopA localization was unaltered in a pleD mutant. Also, PopA localization was not affected in mutants lacking any of the other 12 C. crescentus proteins harboring a GGDEF or EAL domain. This leaves the possibility that several DGCs redundantly contribute to the c-di-GMP pool required for PopA activation, a phenomenon that has been described for several c-diGMP-dependent pathways in different organisms (Simm et al. 2004; Kader et al. 2006; Lim et al. 2006; Weber et al. 2006). Alternatively, cell cycle timing of c-di-GMP levels and PopA localization to the stalked cell pole might ultimately be determined by a c-di-GMP-specific phos- phodiesterase, which specifically reduces c-di-GMP levels in G1. While the additional components of this pathway remain to be identified, this is the first report that links c-di-GMP to the dynamic spatiotemporal control of the bacterial cell cycle. This is reminiscent of the function of eukaryotic second messengers in cell polarity and behavior (Janetopoulos et al. 2005; Evans and Falke 2007; Insall and Andrew 2007).

Two response regulators, CpdR and PopA, are involved in directing the protease ClpXP and its substrate CtrA to the emerging stalked cell pole. Polar recruitment of CpdR depends on its phosphorylation state (Iniesta et al. 2006). In particular, in the presence of $\mathrm{CpdR}_{\mathrm{D} 51 \mathrm{~A}}$, a mutant that cannot be phosphorylated, the proportion of cells with CpdR and ClpX at the cell pole is increased, and, as a result, cellular levels of CtrA are severely reduced (Fig. 8; Iniesta et al. 2006). Expression of $\mathrm{CpdR}_{\mathrm{D} 51 \mathrm{~A}}$ also results in a severe cell morphology and cell division phenotype. The finding that cell morphology, division, and CtrA levels are restored in popA mutant cells expressing $c p d R_{D 51 A}$ argues that this phenotype is a direct consequence of reduced levels of CtrA and possibly additional ClpXP substrates. The latter can be inferred from the observation that cell morphology is not fully restored in cells expressing $c p d R_{D 51 A}$ and a stable variant of CtrA. In contrast, the expression of a PopA I-site mutant that fails to localize to the stalked cell pole fully suppresses the $c p d R_{D 51 A}$ phenotype. Together this argues that the two pathways responsible for the polar localization of substrate and protease converge and together are responsible for the accurate cell cycle timing of CtrA degradation (Fig. 9A). How exactly the CpdR and PopA pathways are interlinked is not clear. Mutants lacking CpdR, in addition to being unable to direct ClpXP to the cell pole, also fail to localize CtrA and show a partial defect in localizing RcdA to the cell pole (Iniesta et al. 2006). Conversely, in $\Delta$ popA mutants, CpdR and ClpX frequently mislocalize. This effect is most pronounced during the G1-to-S transition, when CtrA is being degraded. Together with our finding that CpdR weakly interacts with RcdA in the bacterial two-hybrid system (Supplemental Fig. S4), this might indicate that all of these proteins are part of a macromolecular complex at the cell pole that facilitates recruitment of the degradation machinery in response to the appropriate signals at the correct time in the cell cycle. Alternatively, some of these mislocalization effects 
might be indirect, as $\mathrm{CpdR}_{\mathrm{D} 51 \mathrm{~A}}$ localizes normally to the stalked cell pole even when PopA is absent (Fig. 8). Clearly, more in vivo and in vitro studies are required in order to fully appreciate the exact contribution of all of these players to the timing of CtrA degradation during the G1-to-S transition.

\section{Materials and methods}

Strains, plasmids, and media

The bacterial strains and plasmids used in this study are listed in Supplemental Table S1. C. crescentus strains were grown in peptone yeast extract (PYE), minimal glucose media (M2G) (Ely 1991), or minimal xylose media (M2X) at $30^{\circ} \mathrm{C}$, unless stated otherwise. Where necessary, growth medium was supplemented with $\mathrm{D}$-xylose varying from $0.1 \%$ to $0.3 \%$. Newborn swarmer cells (SW cells) were isolated by Ludox gradient centrifugation (Jenal and Shapiro 1996). Escherichia coli strains were grown in Luria Broth (LB) media. Antibiotics for selection were added to the media where necessary.

\section{Microscopy and flow cytometry}

For fluorescence imaging, cells were placed on a microscope slide layered with a pad of $1 \%$ agarose dissolved in water or in PYE for time-lapse microscopy. An Olympus IX71 microscope equipped with an UPlanSApo $100 \times / 1.40$ Oil objective (Olympus) and a coolSNAP HQ (Photometrics) CCD camera was used to take differential interference contrast (DIC) and fluorescence photomicrographs. FITC filter sets-Ex 490/20 nm, Em 528/38 nm for GFP fluorescence; Ex 500/20 nm, Em 535/30 nm for YFP fluorescence; and Ex436/10, Em 470/30 nm for CFP fluorescence-were used with an exposure time of $1.0 \mathrm{sec}$. Images were processed with softWoRx version 3.3.6 (Applied Precision) and Photoshop CS2 (Adobe) software. Flow cytometry was done as described in the Supplemental Material.

\section{Bacterial two-hybrid analysis}

Proteins of interest were fused in frame to the $3^{\prime}$ end of the T25 fragment (pKT25) and to the $3^{\prime}$ end (pUT18C) or 5' end (pUT18) of the T18 fragment of the $B$. pertussis adenylate cyclase (Karimova et al. 1998). pKT25-zip and pUT18C-zip were used as positive controls. The adenylate cyclase-deficient $E$. coli strain MM337 was used to screen for positive interactions. pKT25 derivatives were transformed together with pUT18 or pUT18C derivatives into MM337 and the transformants selected on LB with ampicillin $(100 \mu \mathrm{g} / \mathrm{mL})$ and kanamycin $(50 \mu \mathrm{g} / \mathrm{mL})$. To screen for protein-protein interaction, single colonies were streaked on McConkey Agar Base supplemented with maltose $(1 \%)$, ampicillin $(100 \mu \mathrm{g} / \mathrm{mL})$, and kanamycin $(50 \mu \mathrm{g} / \mathrm{mL})$.

\section{Protein purification and UV cross-link assay}

Expression plasmids (pET21C; Novagen) were transformed into E. coli BL21 (DE3) ArcticExpress (Stratagen). Expression and purification of PopA wild-type and mutant forms were carried out according to the manufacturers' instructions (see the Supplemental Material). UV cross-link experiments with $\left[{ }^{33} \mathrm{P}\right] \mathrm{c}$-diGMP were done as described (Christen et al. 2006) (see the Supplemental Material). Pulse-chase experiments were done as described in the Supplemental Material.

\section{FRET}

Cultures of strains UJ4329 and UJ4330 were grown in PYE supplemented with $2.5 \mu \mathrm{g} / \mathrm{mL}$ tetracycline and $2.5 \mu \mathrm{g} / \mathrm{mL}$ gentamycin until they reached an $\mathrm{OD}_{660}$ of 0.3 . Five milliliters of this culture were harvested by centrifugation, washed with and resuspended in $50 \mu \mathrm{L}$ of tethering buffer $(10 \mathrm{mM}$ potassium phosphate, $0.1 \mathrm{mM}$ EDTA, $1 \mathrm{mM}$ L-methionine, $10 \mathrm{mM}$ sodium lactate at $\mathrm{pH}$ 7). For FRET analysis, the cell suspension was placed on thin agarose pads ( $1 \%$ agarose in tethering buffer) on microscopy slides and allowed to immobilize for 5-10 $\mathrm{min}$. The agarose pads were then covered with coverslips and were sealed with Apiezon grease. Fluorescence of 300-500 cells was monitored in each experiment. Fluorescence signals in cyan and yellow channels were detected using two photon-counting photomultipliers (H7421-40; Hamamatsu) whose outputs were converted to analog signals by ratemeters (RIS-375; Rowland Institute).

\section{Comparative modeling of PopA}

A comparative 3D model of C. crescentus PopA was built based on the crystal structure of the response regulator PleD in complex with c-di-GMP (PDB: 1W25) (Chan et al. 2004). Template identification and alignment were performed by scanning the PDB database (Berman et al. 2000) for suitable template structures using a PSI-BLAST (Altschul et al. 1997) sequence profile for the target based on the NCBI nonredundant protein sequence database (Wheeler et al. 2007). Model coordinates were generated in Swiss-Model Workspace following visual assessment of placements of insertions and deletions in the alignment (Schwede et al. 2003). The orientation of c-di-GMP in the model was inferred from the PleD-c-di-GMP template structure complex.

\section{Acknowledgments}

We thank A. Iniesta and L. Shapiro for strains, F. Hamburger for construction of plasmids, and Victor Sourjik and Hui Li for their help with FRET analysis. This work was supported by Swiss National Science Foundation Fellowship 3100A0-108186 to U.J.

\section{References}

Alba, B.M., Leeds, J.A., Onufryk, C., Lu, C.Z., and Gross, C.A. 2002. DegS and YaeL participate sequentially in the cleavage of RseA to activate the $\sigma^{\mathrm{E}}$-dependent extracytoplasmic stress response. Genes \& Dev. 16: 2156-2168.

Aldridge, P., Paul, R., Goymer, P., Rainey, P., and Jenal, U. 2003. Role of the GGDEF regulator PleD in polar development of Caulobacter crescentus. Mol. Microbiol. 47: 1695-1708.

Altschul, S.F., Madden, T.L., Schaffer, A.A., Zhang, J., Zhang, Z., Miller, W., and Lipman, D.J. 1997. Gapped BLAST and PSIBLAST: A new generation of protein database search programs. Nucleic Acids Res. 25: 3389-3402.

Berman, H.M., Westbrook, J., Feng, Z., Gilliland, G., Bhat, T.N., Weissig, H., Shindyalov, I.N., and Bourne, P.E. 2000. The Protein Data Bank. Nucleic Acids Res. 28: 235-242.

Biondi, E.G., Reisinger, S.J., Skerker, J.M., Arif, M., Perchuk, B.S., Ryan, K.R., and Laub, M.T. 2006. Regulation of the bacterial cell cycle by an integrated genetic circuit. Nature 444: 899-904.

Chan, C., Paul, R., Samoray, D., Amiot, N.C., Giese, B., Jenal, U., and Schirmer, T. 2004. Structural basis of activity and allosteric control of diguanylate cyclase. Proc. Natl. Acad. Sci. 101: 17084-17089.

Chen, J.C., Hottes, A.K., McAdams, H.H., McGrath, P.T., Viollier, P.H., and Shapiro, L. 2006. Cytokinesis signals 
truncation of the PodJ polarity factor by a cell cycle-regulated protease. EMBO J. 25: 377-386.

Chien, P., Perchuk, B.S., Laub, M.T., Sauer, R.T., and Baker, T.A. 2007. Direct and adaptor-mediated substrate recognition by an essential AAA+ protease. Proc. Natl. Acad. Sci. 104: 65906595.

Christen, M., Christen, B., Folcher, M., Schauerte, A., and Jenal, U. 2005. Identification and characterization of a cyclic diGMP-specific phosphodiesterase and its allosteric control by GTP. J. Biol. Chem. 280: 30829-30837.

Christen, B., Christen, M., Paul, R., Schmid, F., Folcher, M., Jenoe, P., Meuwly, M., and Jenal, U. 2006. Allosteric control of cyclic di-GMP signaling. J. Biol. Chem. 281: 32015-32024.

Domian, I.J., Quon, K.C., and Shapiro, L. 1997. Cell type-specific phosphorylation and proteolysis of a transcriptional regulator controls the G1-to-S transition in a bacterial cell cycle. Cell 90: 415-424.

Domian, I.J., Reisenauer, A., and Shapiro, L. 1999. Feedback control of a master bacterial cell-cycle regulator. Proc. Natl. Acad. Sci. 96: 6648-6653.

Ely, B., 1991. Genetics of Caulobacter crescentus. Methods Enzymol. 204: 372-384.

Evans, J.H., and Falke, J.J. 2007. $\mathrm{Ca}^{2+}$ influx is an essential component of the positive-feedback loop that maintains leading-edge structure and activity in macrophages. Proc. Natl. Acad. Sci. 104: 16176-16181.

Gonzalez, M., Rasulova, F., Maurizi, M.R., and Woodgate, R. 2000. Subunit-specific degradation of the UmuD/D' heterodimer by the ClpXP protease: The role of trans recognition in UmuD' stability. EMBO J. 19: 5251-5258.

Gottesman, S., 2003. Proteolysis in bacterial regulatory circuits. Annu. Rev. Cell Dev. Biol. 19: 565-587.

Grünenfelder, B., Rummel, G., Vohradsky, J., Röder, D., Langen, H., and Jenal, U. 2001. Proteomic analysis of the bacterial cell cycle. Proc. Natl. Acad. Sci. 98: 4681-4686.

Hinz, A.J., Larson, D.E., Smith, C.S., and Brun, Y.V. 2003. The Caulobacter crescentus polar organelle development protein PodJ is differentially localized and is required for polar targeting of the PleC development regulator. Mol. Microbiol. 47: 929-941.

Iniesta, A.A., McGrath, P.T., Reisenauer, A., McAdams, H.H., and Shapiro, L. 2006. A phospho-signaling pathway controls the localization and activity of a protease complex critical for bacterial cell cycle progression. Proc. Natl. Acad. Sci. 103: 10935-10940.

Insall, R., and Andrew, N. 2007. Chemotaxis in Dictyostelium: How to walk straight using parallel pathways. Curr. Opin. Microbiol. 10: 578-581.

Janetopoulos, C., Borleis, J., Vazquez, F., Iijima, M., and Devreotes, P. 2005. Temporal and spatial regulation of phosphoinositide signaling mediates cytokinesis. Dev. Cell 8: 467477.

Jenal, U. and Fuchs, T. 1998. An essential protease involved in bacterial cell-cycle control. EMBO J. 17: 5658-5669.

Jenal, U. and Hengge-Aronis, R. 2003. Regulation by proteolysis in bacterial cells. Curr. Opin. Microbiol. 6: 163-172.

Jenal, U. and Malone, J. 2006. Mechanisms of cyclic-di-GMP signaling in bacteria. Annu. Rev. Genet. 40: 385-407.

Jenal, U. and Shapiro, L. 1996. Cell cycle-controlled proteolysis of a flagellar motor protein that is asymmetrically distributed in the Caulobacter predivisional cell. EMBO I. 15: 2393-2406.

Johansson, U. and Uhlin, B..E. 1999. Differential proteasemediated turnover of H-NS and StpA revealed by a mutation altering protein stability and stationary-phase survival of Escherichia coli. Proc. Natl. Acad. Sci. 96: 10776-10781.
Kader, A., Simm, R., Gerstel, U., Morr, M., and Romling, U. 2006. Hierarchical involvement of various GGDEF domain proteins in rdar morphotype development of Salmonella enterica serovar Typhimurium. Mol. Microbiol. 60: 602616.

Kanehara, K., Ito, K., and Akiyama, Y. 2002. YaeL (EcfE) activates the $\sigma^{\mathrm{E}}$ pathway of stress response through a site-2 cleavage of anti- $\sigma^{\mathrm{E}}$, RseA. Genes \& Dev. 16: 2147-2155.

Karimova, G., Pidoux, J., Ullmann, A., and Ladant, D. 1998. A bacterial two-hybrid system based on a reconstituted signal transduction pathway. Proc. Natl. Acad. Sci. 95: 5752-5756.

Keiler, K.C., Waller, P.R., and Sauer, R.T. 1996. Role of a peptide tagging system in degradation of proteins synthesized from damaged messenger RNA. Science 271: 990-993.

Kim, D.Y. and Kim, K..K. 2003. Crystal structure of ClpX molecular chaperone from Helicobacter pylori. I. Biol. Chem. 278: 50664-50670.

Kulasakara, H., Lee, V., Brencic, A., Liberati, N., Urbach, J., Miyata, S., Lee, D.G., Neely, A.N., Hyodo, M., Hayakawa Y., et al. 2006. Analysis of Pseudomonas aeruginosa diguanylate cyclases and phosphodiesterases reveals a role for bis- $\left(3^{\prime}-5^{\prime}\right)$ cyclic-GMP in virulence. Proc. Natl. Acad. Sci. 103: 28392844.

Lawler, M.L., Larson, D.E., Hinz, A.J., Klein, D., and Brun, Y.V. 2006. Dissection of functional domains of the polar localization factor PodJ in Caulobacter crescentus. Mol. Microbiol. 59: 301-316.

Levi, A. and Jenal, U. 2006. Holdfast formation in motile Swarmer cells optimizes surface attachment during Caulobacter crescentus development. J. Bacteriol. 188: 5315-5318.

Lim, B., Beyhan, S., Meir, J., and Yildiz, F.H. 2006. Cyclic-diGMP signal transduction systems in Vibrio cholerae: Modulation of rugosity and biofilm formation. Mol. Microbiol. 60: 331-348.

Malone, J.G., Williams, R., Christen, M., Spiers, A.J., Jenal, U., and Rainey, P.B. 2006. The structure-function relationship of WspR; a Pseudomonas fluorescens response regulator with a GGDEF output domain. Microbiology 153: 980-994.

McGrath, P.T., Iniesta, A.A., Ryan, K.R., Shapiro, L., and McAdams, H.H. 2006. A dynamically localized protease complex and a polar specificity factor control a cell cycle master regulator. Cell 124: 535-547.

Miyawaki, A., and Tsien, R.Y. 2000. Monitoring protein conformations and interactions by fluorescence resonance energy transfer between mutants of green fluorescent protein. Methods Enzymol. 327: 472-500.

Paul, R., Weiser, S., Amiot, N.C., Chan, C., Schirmer, T., Giese, B., and Jenal, U. 2004. Cell cycle-dependent dynamic localization of a bacterial response regulator with a novel diguanylate cyclase output domain. Genes \& Dev. 18: 715-727.

Paul, R., Abel, S., Wassmann, P., Beck, A., Heerklotz, H., and Jenal, U. 2007. Activation of the diguanylate cyclase pleD by phosphorylation-mediated dimerization. J. Biol. Chem. 282: 29170-29177.

Paul, R., Jaeger, T., Abel, S., Wiederkehr, I., Folcher, M., Biondi, E.G., Laub, M.T., and Jenal, U. 2008. Allosteric regulation of histidine kinases by their cognate response regulator determines cell fate. Cell 133: 452-461.

Quon, K.C., Yang, B., Domian, I.J., Shapiro, L., and Marczynski, G.T. 1998. Negative control of bacterial DNA replication by a cell cycle regulatory protein that binds at the chromosome origin. Proc. Natl. Acad. Sci. 95: 120-125.

Ryan, K.R., Judd, E.M., and Shapiro, L. 2002. The CtrA response regulator essential for Caulobacter crescentus cell-cycle progression requires a bipartite degradation signal for temporally controlled proteolysis. J. Mol. Biol. 324: 443-455. 
Ryan, K.R., Huntwork, S., and Shapiro, L. 2004. Recruitment of a cytoplasmic response regulator to the cell pole is linked to its cell cycle-regulated proteolysis. Proc. Natl. Acad. Sci. 101: 7415-7420.

Schwede, T., Kopp, J., Guex, N., and Peitsch, M.C. 2003. SWISSMODEL: An automated protein homology-modeling server. Nucleic Acids Res. 31: 3381-3385.

Selvin, P.R., 2000. The renaissance of fluorescence resonance energy transfer. Nat. Struct. Biol. 7: 730-734.

Simm, R., Morr, M., Kader, A., Nimtz, M., and Romling, U. 2004. GGDEF and EAL domains inversely regulate cyclic diGMP levels and transition from sessility to motility. Mol. Microbiol. 53: 1123-1134.

Sourjik, V., and Berg, H.C. 2002. Receptor sensitivity in bacterial chemotaxis. Proc. Natl. Acad. Sci. 99: 123-127.

Tamayo, R., Pratt, J.T., and Camilli, A. 2007. Roles of cyclic diguanylate in the regulation of bacterial pathogenesis. Annu Rev Microbiol. 61: 131-148.

Thanbichler, M., Iniesta, A.A., and Shapiro, L. 2007. A comprehensive set of plasmids for vanillate- and xylose-inducible gene expression in Caulobacter crescentus. Nucleic Acids Res. 35: e137. doi:10.1093/nar/gkm818.

Tsai, J.W. and Alley, M.R. 2001. Proteolysis of the Caulobacter McpA chemoreceptor is cell cycle regulated by a ClpXdependent pathway. J. Bacteriol. 183: 5001-5007.

Turgay, K., Hahn, J., Burghoorn, J., and Dubnau, D. 1998. Competence in Bacillus subtilis is controlled by regulated proteolysis of a transcription factor. EMBO J. 17: 6730-6738.

Viollier, P.H., Sternheim, N., and Shapiro, L. 2002. Identification of a localization factor for the polar positioning of bacterial structural and regulatory proteins. Proc. Natl. Acad. Sci. 99: 13831-13836.

Wang, S.P., Sharma, P.L., Schoenlein, P.V., and Ely, B. 1993. A histidine protein kinase is involved in polar organelle development in Caulobacter crescentus. Proc. Natl. Acad. Sci. 90: 630-634.

Wang, J., Hartling, J.A., and Flanagan, J.M. 1998. Crystal structure determination of Escherichia coli ClpP starting from an EM-derived mask. J. Struct. Biol. 124: 151-163.

Wassmann, P., Chan, C., Paul, R., Beck, A., Heerklotz, H., Jenal, U., and Schirmer, T. 2007. Structure of BeF-modified response regulator PleD: Implications for diguanylate cyclase activation, catalysis, and feedback inhibition. Structure 15: 915-927.

Weber, H., Pesavento, C., Possling, A., Tischendorf, G., and Hengge, R. 2006. Cyclic-di-GMP-mediated signaling within the $\sigma^{\mathrm{s}}$ network of Escherichia coli. Mol. Microbiol. 62: 10141034.

Wheeler, D.L., Barrett, T., Benson, D.A., Bryant, S.H., Canese, K. Chetvernin, V., Church, D.M., DiCuccio, M., Edgar, R., and Federhen, S., et al. 2007. Database resources of the National Center for Biotechnology Information. Nucleic Acids Res. 35: D5-D12. doi:10.1093/nar/gki062.

Zhu, J. and Winans, S.C. 2001. The quorum-sensing transcriptional regulator TraR requires its cognate signaling ligand for protein folding, protease resistance, and dimerization. Proc. Natl. Acad. Sci. 98: 1507-1512. 


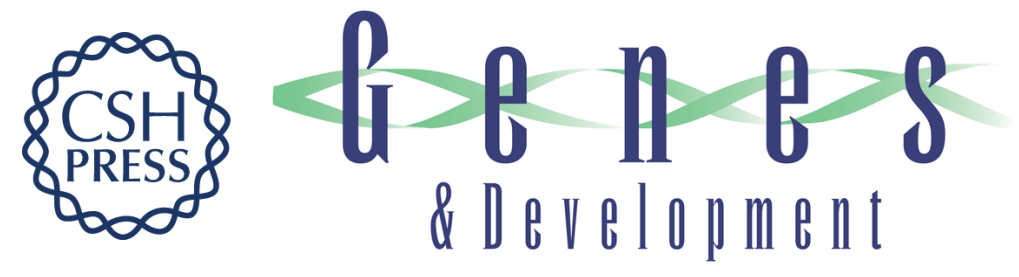

\section{Second messenger-mediated spatiotemporal control of protein degradation regulates bacterial cell cycle progression}

Anna Duerig, Sören Abel, Marc Folcher, et al.

Genes Dev. 2009, 23:

Access the most recent version at doi:10.1101/gad.502409

Supplemental http://genesdev.cshlp.org/content/suppl/2009/01/07/23.1.93.DC1
Material

References This article cites 59 articles, 28 of which can be accessed free at:

http://genesdev.cshlp.org/content/23/1/93.full.html\#ref-list-1

License

Email Alerting Receive free email alerts when new articles cite this article - sign up in the box at the top

Service right corner of the article or click here.

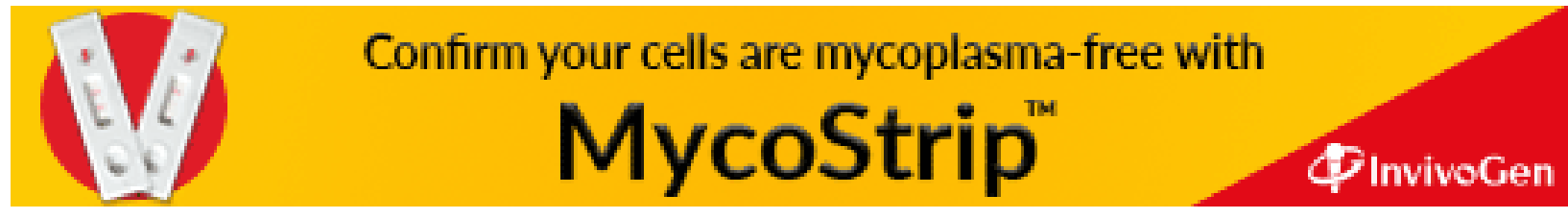

